\title{
ScienceDirect
}

Contents lists available at sciencedirect.com

Journal homepage: www.elsevier.com/locate/jval

\section{Cost-Effectiveness Analysis of a National Pre-Exposure Prophylaxis (PrEP) Program in Ireland}

Eamon O Murchu, MB BCh, MPH, Conor Teljeur, PhD, Catherine Hayes, MB, MPH, Patricia Harrington, PhD, Patrick Moran, PhD, Máirín Ryan, $\mathrm{PhD}$

\section{A B S T R A C T}

Objectives: To estimate the cost-effectiveness of introducing a publicly funded pre-exposure prophylaxis (PrEP) program in Ireland.

Methods: We constructed a state-transition Markov model. This was a cross-sectional population model that tracked all HIVnegative men who have sex with men (MSM) in Ireland over their lifetime. Access to a publicly funded PrEP program (medications + frequent monitoring) in high-risk MSM was compared with no PrEP. The primary outcome measure was the incremental cost-effectiveness ratio (ICER).

Results: In the base case, introducing a PrEP program was considered cost saving and provided significant health benefits to the population. Univariate sensitivity analysis demonstrated that PrEP efficacy and HIV incidence had the greatest impact on cost-effectiveness. Including an increase in sexually transmitted infections had a negligible impact on the results.

Efficacy was a significant driver in the model. PrEP was cost saving at all efficacy values above $60 \%$, and at the lowest reported efficacy in MSM (44\% in the iPrEX trial), the ICER was $€ 4711 /$ QALY (highly cost-effective). Event-based dosing (administration during high-risk periods only) was associated with additional cost savings.

We estimated that 1705 individuals (95\% CI: 617-3452) would join the program in year 1 . The incremental budget impact was $€ 1.5 \mathrm{~m}(95 \% \mathrm{CI}: € 0.5 \mathrm{~m}$ to $€ 3 \mathrm{~m})$ in the first year and $€ 5.4 \mathrm{~m}$ over 5 years ( $95 \% \mathrm{CI}: € 1.8 \mathrm{~m}$ to $€ 11.5 \mathrm{~m}$ ), with 173 cases of HIV averted over 5 years.

Conclusion: We found that the introduction of a PrEP program would be considered cost saving in the first cost-effectiveness analysis of its kind in Ireland.

Keywords: cost-effectiveness analysis, HIV prevention, pre-exposure prophylaxis, PrEP

VALUE HEALTH. 2021;

\section{Introduction}

HIV infection remains a major global public health threat. In Ireland, 523 diagnoses of HIV were notified in 2018, representing a rate of 11.0 per 100000 population, ${ }^{1}$ higher than the European average (5.6 per $100000^{2}$ ). Men who have sex with men (MSM) accounted for $56 \%$ of all infections, whereas this group only accounts for approximately $4 \%$ of the overall male population.

Pre-exposure prophylaxis (PrEP) is a form of HIV prevention whereby antiretrovirals are taken by HIV-negative individuals at high sexual risk to prevent infection. PrEP is safe and highly effective, especially in the MSM group ( $86 \%$ effectiveness ${ }^{4,5}$ ). Once daily oral tenofovir/emtricitabine was first licensed for use as PrEP in 2016 in Ireland. Ideally, PrEP should be provided as part of a dedicated program that includes frequent testing for HIV and other sexually transmitted infections (STIs), safer sex counseling, and medication adherence support.

Significant variation in the cost-effectiveness of PrEP exists in the international literature. ${ }^{6}$ Similar to most interventions, the cost-effectiveness of PrEP is affected by a number of parameters that are often country specific, such as the cost of PrEP. In recent years, multiple generic tenofovir/emtricitabine manufacturers have entered the Irish market, reducing medication costs by over $90 \%$ (a 30-day supply of generic PrEP is now approximately $€ 50$ ). ${ }^{6}$ The objective of this study was to estimate the cost-effectiveness of a PrEP program for MSM at high risk in Ireland. This costeffectiveness analysis was previously published as part of a larger health technology assessment by the Health Information and Quality Authority and made available on the Health Information and Quality Authority website. ${ }^{7}$ The results of this study were expected to inform a decision by the Irish Department of Health on whether or not to introduce a national, publicly funded PrEP program.

\section{Methods}

We conducted a full economic evaluation to estimate the costs and consequences of introducing a PrEP program for MSM at high 
risk in Ireland, following national ${ }^{8}$ and international ${ }^{9}$ methodological guidelines. Our primary outcome was the incremental costeffectiveness ratio (ICER). The willingness-to-pay (WTP) threshold in Ireland is $€ 45000$ per quality-adjusted life-year (QALY) gained.

\section{Intervention and Comparator}

The intervention comprised free access to a publicly funded PrEP program (daily oral tenofovir/emtricitabine and 3-monthly STI clinic visits that include HIV/STI testing and renal function monitoring) for MSM at high risk. The comparator was standard care (the current suite of HIV prevention strategies), without access to a dedicated PrEP program. We did not model PrEP administration to MSM in medium-/low-risk groups, or to all MSM, as these individuals are not eligible for PrEP (tenofovir/ emtricitabine is only licensed for use in individuals at high risk, and high PrEP efficacy has only been observed in high-risk groups $^{4,5}$ ).

\section{Target Population}

We included all HIV-negative MSM in Ireland in the model. The eligibility criteria for PrEP defined our high-risk group (based national clinical guidelines, see Appendix 1 in Supplemental Materials found at https://doi.org/10.1016/j.jval.2021.02.005). Briefly, MSM who satisfy any of the following criteria are eligible:

- $\geq 2$ episodes of condomless anal sex in the past 6 months

- acute bacterial STI diagnosis in the past 12 months

- post-exposure prophylaxis following sexual exposure (PEPSE) in the past 12 months

- sex under the influence of drugs in the past 6 months

We determined the size of the target population ( $\mathrm{N}=1705 \operatorname{PrEP}$ users in year 1 , mean age 36.7 ) using 4 probability distributions: the proportion of all men who are MSM, the proportion of MSM who are sexually active, the proportion of sexually active MSM who are considered high risk, and the program uptake rate (see Appendix 2.1 in Supplemental Materials found at https://doi.org/1 0.1016/j.jval.2021.02.005).

\section{Model Structure}

We developed a state-transition Markov model. The model was a closed cross-sectional population model that tracked the entire population of HIV-negative MSM in Ireland at the outset of the simulation (2018) and followed these men over their lifetime. A limitation of our choice of model is that it does not contain dynamic elements. Therefore, the model underestimates the net benefit, because it does not capture the indirect reduction in onward HIV transmission associated with PrEP use. All analyses were performed using TreeAge Pro 2018. ${ }^{10}$

The model contains 5 health states (Fig. 1). The first 3 health states encompass all HIV-negative MSM: high-risk MSM taking PrEP (PrEP high risk), high-risk MSM not taking PrEP (no PrEP high risk), and all other MSM (medium/low risk). The eligibility criteria for PrEP defined the high-risk group. We assumed that PrEP would not be taken by individuals in the medium/low-risk group, because they are not eligible. Sexual mixing was possible between risk groups. Behavior change was accounted for; those in the medium-/low-risk group could transition to high risk over time (and vice versa), until they acquire HIV or die (transition probability distributions are provided in Appendix 5 in Supplemental Materials found at https://doi.org/10.1016/j.jval.2021.02.005).

We adopted the perspective of the Health Service Executive, the body responsible for public health and social care services in Ireland. We only considered direct costs, and the time horizon of
Figure 1. The basic model structure is shown. There are 5 health states. Risk status of HIV negative MSM is either categorized as "high" or "medium/low" risk. High risk is defined by the eligibility criteria for PrEP. All others are considered medium/low risk. Additionally, high-risk individuals may be taking PrEP (PrEP high risk) or not (No PrEP high risk). Individuals may move between these health states, until they acquire HIV or die.

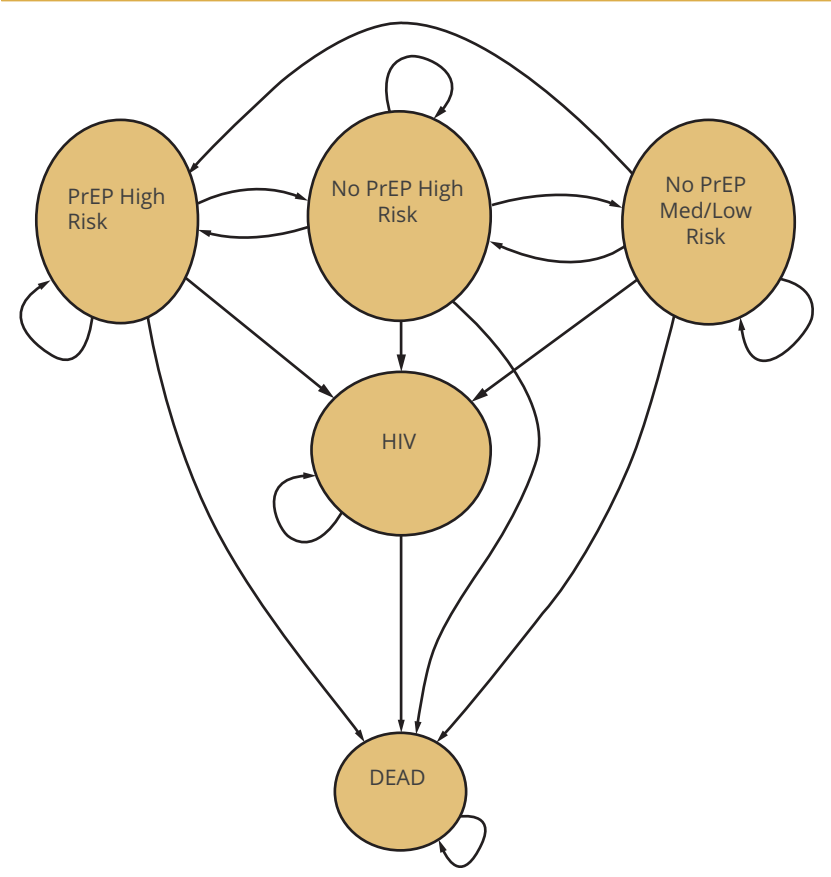

PrEP indicates pre-exposure prophylaxis.

the analysis was the cohort's lifetime. We applied a 5.0\% discount rate to costs and benefits.

\section{Model Assumptions}

We only modeled MSM, based on the assumption that over 95\% of prospective PrEP users will belong to this group (for comparison, over 99\% of participants in Scotland's first year of PrEP were MSM $^{11}$ ).

We assumed that only MSM at high risk would be offered PrEP (according to the PrEP eligibility criteria). We also assumed that individuals taking PrEP whose risk status changes to medium/low risk would stop taking PrEP from their next 3 monthly clinic visits onward (as they are no longer eligible for PrEP). PrEP retention rates were only identified in 1 study from Australia $^{12}$ : a 76\% (95\% confidence interval [CI]: 74-77) 1-year retention rate was applied to the model (median duration of PrEP use $=2.5$ years).

We assumed that antiretroviral therapy starts immediately after HIV diagnosis, in line with current best practice. ${ }^{13}$ There is often a delay between infection and diagnosis, however. As PrEP users attend 3-monthly appointments, we assumed that the delay from infection to diagnosis and treatment would not extend beyond 1 cycle ( 1 year) in the model.

\section{Clinical and Epidemiological Parameter Estimates}

We obtained epidemiological parameters (Table 1) from national reporting bodies (the Central Statistics Office ${ }^{14}$ and the Health Protection Surveillance Centre $^{15}$ ). In addition, we 
Table 1. Epidemiological parameter estimates.

\begin{tabular}{|c|c|c|c|}
\hline Epidemiological parameter & Value & $95 \% \mathrm{Cl}$ & Source \\
\hline \multicolumn{4}{|l|}{ Target population } \\
\hline Population of Ireland & 4857015 & & CSO 2018 population estimates \\
\hline Male population aged 16 to 80 & 1802395 & & CSO 2018 population estimates \\
\hline Proportion MSM & $5 \%$ & $4-6$ & Healthy Ireland surveys $2015^{35}$ and $2017^{3}$ \\
\hline MSM population & 84713 & 68 491-101 737 & \\
\hline Proportion MSM sexually active & $63 \%$ & 49-76 & $\begin{array}{l}\text { Pooled data from } 2 \text { surveys }{ }^{27,36} \text { (see } \\
\text { Appendix } 2.1 .2 \text { in Supplemental Materials } \\
\text { found at https://doi.org/10.1016/j.jval.2 } \\
021.02 .005 \text { ) }\end{array}$ \\
\hline $\begin{array}{l}\text { Proportion MSM sexually active at high } \\
\text { sexual risk for HIV }\end{array}$ & $23 \%$ & $22.7-23.3$ & $\begin{array}{l}\text { MISI } 2015^{25} \text { (see Appendix } 2.1 .3 \text { in } \\
\text { Supplemental Materials found at https:// } \\
\text { doi.org/10.1016/j.jval.2021.02.005) }\end{array}$ \\
\hline $\begin{array}{l}\text { Target population (sexually active MSM at } \\
\text { high risk) }\end{array}$ & 12275 & $7618-18106$ & Calculation \\
\hline \multicolumn{4}{|l|}{ HIV incidence } \\
\hline Overall incidence among MSM & 2 per 100 person-years & 1.8-2.2 per 100 person-years & Ong et al., $2017^{26}$ \\
\hline Incidence in high risk stratum of MSM & 3.3 per 100 person-years & 2.8-4.9 per 100 person-years & Ong et al., $2017^{26}$ \\
\hline \multicolumn{4}{|l|}{ Clinical Effectiveness } \\
\hline Efficacy of PrEP to prevent HIV & $75 \%$ & $49-90$ & Meta-analysis of 6 trials ${ }^{4,5,18-21}$ \\
\hline $\begin{array}{l}\text { Efficacy of PrEP to prevent HIV in high } \\
(>80 \% \text { adherence) trials }\end{array}$ & $86 \%$ & $65-94$ & Meta-analysis of 2 trials ${ }^{4,5}$ \\
\hline \multicolumn{4}{|l|}{ Increased mortality associated with HIV } \\
\hline Standardized mortality ratio & 5.7 & $5.5-5.8$ & Croxford et al. $2017^{16}$ \\
\hline \multicolumn{4}{|l|}{ Disutilities owing to HIV positivity } \\
\hline HIV + & -0.11 & -0.13 to -0.10 & Miners et al. $2014^{17}$ \\
\hline
\end{tabular}

conducted a systematic review of prior cost-effectiveness studies that contributed parameter data (see Appendix 2.4 in Supplemental Materials found at https://doi.org/10.1016/j.jval.2021.02. 005). We applied international data in cases where Irish data were unavailable, such as the loss of utility and mortality associated with HIV infection (retrieved from 2 UK studies ${ }^{16,17}$ ).

We previously conducted a systematic review to obtain estimates of the clinical effectiveness of PrEP. ${ }^{6}$ The measure of effectiveness in MSM was derived from a meta-analysis of 6 trials. ${ }^{4,5,18-21}$

\section{Cost Estimates}

We employed a microcosting approach to estimate the cost of PrEP, PEPSE, STI clinic visits, laboratory tests, and treatment of STIs (Table 2, and Appendix 2.3 in Supplemental Materials found at https://doi.org/10.1016/j.jval.2021.02.005). We used UK data to estimate incremental lifetime treatment costs in HIV-positive individuals owing to the absence of Irish data. Analysis was performed from a healthcare perspective, consistent with national health technology assessment guidelines. ${ }^{8}$ We inflated retrospective costs using the Irish Consumer Price Index for health.

\section{Quality of Life and Utility Estimates}

We obtained baseline quality-of-life estimates for men by age from UK estimates for a general population, in the absence of validated Irish data. ${ }^{22}$ We obtained utility weights for HIV from a 2014 study, and adjusted by age. ${ }^{17}$ We assumed that there is no utility loss for undiagnosed HIV-positive individuals.

\section{Process for Calibrating Model}

Very limited Irish data were available for a number of model parameters, and others were based on international estimates that may not be directly applicable to Ireland. We therefore used a calibration exercise to investigate which parameter values would lead to plausible results for 2 model outputs: (1) HIV incidence in MSM, and (2) the size of the program. We considered that between 150 and 400 HIV cases in MSM per year was plausible (based on Health Protection Surveillance Centre data ${ }^{15,23}$ ), and we considered that the enrollment of 1000 to 3000 individuals in first year was plausible. The only data to inform this range came from the PrEP program in Scotland, the first program to report national data following PrEP implementation. ${ }^{11}$ In its first year of operation, 1872 people availed of PrEP (and Scotland's population is approximately $10 \%$ larger than Ireland).

We used both Latin Hypercube and Monte Carlo parameter value sampling (see Appendix 3 in Supplemental Materials found at https://doi.org/10.1016/j.jval.2021.02.005). After calibration, we refitted 6 model parameters (Table 3).

\section{Risk Compensation and Potential Increase in STIS}

PrEP offers no protection against any infection other than HIV. A rise in STI diagnoses following PrEP introduction may be a result of a real increase in STI transmission, or may simply reflect improved detection owing to the frequent testing as part of the 
Table 2. Cost parameter estimates.

\begin{tabular}{|c|c|c|}
\hline Cost parameter & Value & Source \\
\hline \multicolumn{3}{|l|}{ Medication-only costs } \\
\hline Yearly cost of PrEP, per patient & $€ 638$ & $\begin{array}{l}\text { Personal communication (Appendix 2.3.1 in Supplemental } \\
\text { Materials found at https://doi.org/10.1016/j.jval.2021.02.005) }\end{array}$ \\
\hline \multicolumn{3}{|l|}{ Program-only costs } \\
\hline First assessment & $€ 187$ & $\begin{array}{l}\text { Microcosting (Appendix 2.3.2 in Supplemental Materials } \\
\text { found at https://doi.org/10.1016/j.jval.2021.02.005) }\end{array}$ \\
\hline Starting visit* & $€ 16$ & $\begin{array}{l}\text { Microcosting (Appendix 2.3.2 in Supplemental Materials } \\
\text { found at https://doi.org/10.1016/j.jval.2021.02.005) }\end{array}$ \\
\hline Subsequent visits in year 1 & $€ 118$ & $\begin{array}{l}\text { Microcosting (Appendix 2.3.2 in Supplemental Materials } \\
\text { found at https://doi.org/10.1016/j.jval.2021.02.005) }\end{array}$ \\
\hline Total (first assessment, starting visit, 3 subsequent visits) & $€ 550$ & $\begin{array}{l}\text { Microcosting (Appendix 2.3.2 in Supplemental Materials } \\
\text { found at https://doi.org/10.1016/j.jval.2021.02.005) }\end{array}$ \\
\hline \multicolumn{3}{|l|}{ Cost of usual care } \\
\hline Clinic visits for high-risk MSM, per patient & $€ 127$ & $\begin{array}{l}\text { Microcosting (see Appendix 2.3.2 in Supplemental Materials } \\
\text { found at https://doi.org/10.1016/j.jval.2021.02.005) }\end{array}$ \\
\hline Average yearly realized cost, per patient & $€ 285$ & $\begin{array}{l}\text { Assumption ( } 25 \% \text { unengaged, } 37.5 \% \text { attend } 4 \text { visits/year and } \\
37.5 \% \text { attend } 2 \text { visits/year) }\end{array}$ \\
\hline \multicolumn{3}{|l|}{ Incremental costs } \\
\hline Incremental cost of program (per PrEP user per year) & \multicolumn{2}{|l|}{$€ 265$} \\
\hline $\begin{array}{l}\text { Incremental cost of program + PrEP medications (per PrEP } \\
\text { user per year) }\end{array}$ & $€ 903$ & \\
\hline \multicolumn{3}{|l|}{ HIV costs } \\
\hline $\begin{array}{l}\text { Mean undiscounted lifetime costs associated with HIV } \\
\text { infection (ART initiated soon after HIV diagnosis) })^{\dagger} \text { per } \\
\text { person }\end{array}$ & $\begin{array}{l}€ 423200 \text { (range: } \\
€ 158200-€ 467 \\
500)\end{array}$ & Nakagawa et al. $2015^{37 \dagger}$ \\
\hline \multicolumn{3}{|l|}{ Additional costs } \\
\hline Cost of 1 course of PrEP following sexual exposure & $€ 964$ & $\begin{array}{l}\text { Microcosting (see Appendix 2.3.4 in Supplemental Materials } \\
\text { found at https://doi.org/10.1016/j.jval.2021.02.005) }\end{array}$ \\
\hline Cost to treat 1 episode of rectal chlamydia & $€ 125$ & $\begin{array}{l}\text { Microcosting (see Appendix 2.3.5 in Supplemental Materials } \\
\text { found at https://doi.org/10.1016/j.jval.2021.02.005) }\end{array}$ \\
\hline \multicolumn{3}{|c|}{$\begin{array}{l}\text { Note. All costs rounded to nearest Euro. } \\
\text { CI indicates confidence interval; MSM, men who have sex with men; PrEP, pre-exposure prophylaxis. } \\
\text { *Approximately } 50 \% \text { of participants will require this additional visit. } \\
{ }^{\dagger} \text { All UK costs converted to Euros. Undiscounted costs from Nakagawa } 2015 \text { subsequently discounted at } 5.0 \% \text { per annum in model. }\end{array}$} \\
\hline
\end{tabular}

program. Health authorities in Scotland have not concluded that PrEP resulted in an increase in STIs. ${ }^{11}$

Some evidence has emerged from observational studies, however, that taking PrEP may result in risk compensation and an increase the transmission of STIs. One meta-analysis by Traeger et al. reported an increase in rectal chlamydia after the introduction of PrEP (odds ratio [OR] 1.59; 95\% CI 1.19-2.13). ${ }^{24}$ An increase in syphilis or gonorrhea (at any site), or chlamydia at nonrectal sites, was not found. Taking a conservative approach, we applied an increase in rectal chlamydia to the model (converting ORs to relative risk, PrEP users would experience an annual increase in rectal chlamydia diagnoses of $33 \%$ ).

\section{Post-Exposure Prophylaxis After Sexual Exposure (PEPSE)}

The overall frequency of PEPSE prescribing is unknown in Ireland. One Irish survey (MISI 2015) reported that of all HIVnegative respondents, $4 \%$ had ever used PEPSE. ${ }^{25}$ Additionally, a UK study found that in the year 2012, 4.48\% of high-risk MSM had used PEPSE. ${ }^{26}$ We therefore applied an annual PEPSE use of $4 \%$ in those not taking PrEP in the model.

\section{Sensitivity Analysis}

All parameters were included in the probabilistic sensitivity analysis, and sampled them from their range of plausible values. A number of parameters were associated with significant uncertainty (in particular, probability distributions associated with the size of the target population and movement between risk groups [see Appendix 5.1 in Supplemental Materials found at https://doi. org/10.1016/j.jval.2021.02.005]). We investigated the effect of uncertainty pertaining to individual parameter estimates through univariate sensitivity analysis. We also carried out a 2-way sensitivity analysis relating to the size of the population (proportion high risk and uptake rate), because these parameters were subject to significant uncertainty.

We performed 2 scenario analyses. In the first, we applied event-based dosing ${ }^{5}$ (PrEP is administered during high-risk 
Table 3. Initial and refit parameter values based on Latin Hypercube sampling.

\begin{tabular}{|c|c|c|c|c|}
\hline \multicolumn{5}{|l|}{ Initial parameter values } \\
\hline Parameter & Distribution & Mean & LCI & UCl \\
\hline Proportion of male population aged $16-80$ who are MSM & Beta & 0.05 & .04 & .06 \\
\hline Proportion of MSM currently sexually active & Beta & 0.6304 & .4877 & .7623 \\
\hline Proportion of MSM population eligible for PrEP & Beta & 0.2000 & .0280 & .4827 \\
\hline Proportion of the PrEP eligible population who are likely to enroll & Beta & 0.2139 & .1396 & .2991 \\
\hline Rate of HIV acquisition in MSM at high risk of HIV acquisition & Gamma & 0.0322 & .0143 & .0573 \\
\hline Rate of HIV acquisition in MSM population at medium/low risk of HIV acquisition & Gamma & 0.0043 & .0015 & .0087 \\
\hline \multicolumn{5}{|l|}{ Refit parameter values } \\
\hline Parameter & Distribution & Mean & LCI & $\mathrm{UCl}$ \\
\hline Proportion of male population aged $16-80$ who are MSM & Beta & 0.0458 & .0368 & .0556 \\
\hline Proportion of MSM currently sexually active & Beta & 0.6091 & .4660 & .7369 \\
\hline Proportion of MSM population eligible for PrEP & Beta & 0.1221 & .049 & .2128 \\
\hline Proportion of the PrEP eligible population who are likely to enroll & Beta & 0.2732 & .1984 & .3575 \\
\hline Rate of HIV acquisition in MSM at high risk of HIV acquisition & Gamma & 0.0300 & .0244 & .0358 \\
\hline Rate of HIV acquisition in MSM population at medium/low risk of HIV acquisition & Gamma & 0.0030 & .0024 & .0036 \\
\hline
\end{tabular}

$\mathrm{Cl}$ indicates confidence interval; HIV, human immunodeficiency virus; LCI, lower confidence interval; MSM, men who have sex with men; PrEP, pre-exposure prophylaxis; UCl, upper confidence interval.

periods only, instead of daily use; see Appendix 1 in Supplemental Materials found at https://doi.org/10.1016/j.jval.2021.02.005). In the second, we modeled a baseline PrEP use in the population, because there is evidence some individuals are already purchasing PrEP in community pharmacies or ordering online. We arbitrarily applied a baseline 10\% PrEP use in high-risk MSM (paid for out of pocket) to both the intervention and comparator groups for this comparison (in the absence of data on the size of this group).

\section{Results}

\section{Base-Case Analysis}

Monte Carlo simulation was performed over the course of 10 000 replications to derive estimates of the costs and consequences of implementing a PrEP program. As we achieved stable ICER estimates after approximately 2000 replications, we assumed that 10000 replications were sufficient to obtain stable results from the probabilistic analysis.

In the base case, we found that PrEP was cost saving (Fig. 2). On a population level (across all risk groups), the mean incremental benefit per MSM was 0.03 QALYs and the incremental cost was - $€ 85$ (providing access to a PrEP program for MSM at high risk is less costly, and more effective, than not providing access). Including a potential increase in STIs in the model had a minimal impact on the ICER.

Based on the cost-effectiveness acceptability curve, we estimated that PrEP has a 94\% probability at a WTP of $€ 20000 /$ QLY and an $87 \%$ probability of being considered cost-effective at $€ 5000 /$ QLY (see Appendix 4 in Supplemental Materials found at https://doi.org/10.1016/j.jval.2021.02.005).

We estimated that, on average, 1705 MSM (95\% CI: 617-3452) would to join the program in year 1 , with a mean age of 36.7 . The incremental budget impact was approximately $€ 1.5 \mathrm{~m}(95 \% \mathrm{CI}$ : $€ 0.5 \mathrm{~m}-€ 3 \mathrm{~m})$ in year 1 and $€ 5.4 \mathrm{~m}(95 \% \mathrm{CI}: € 1.8 \mathrm{~m}-€ 11.5 \mathrm{~m})$ over 5 years. In terms of the effect of PrEP on HIV transmission, we estimated that 173 HIV infections would be averted over 5 years. When we extended the incremental budget impact beyond 5 years, the annual budget impact became cost saving in year 8 . By year 14, the aggregate budget impact became cost saving (breakeven point-all program and medication costs will have been recovered).

\section{Sensitivity Analysis}

We carried out univariate sensitivity analysis to investigate how much uncertainty in the ICER was induced by uncertainty in individual parameters. We varied the efficacy of PrEP from the lowest reported (iPrEX trial, $44 \%)^{18}$ to the highest (PROUD/IPERGAY trials, $86 \%),{ }^{4,5}$ and we varied costs by $20 \%$. Most ICERs remained cost saving (Fig. 3 ; note that variables with $<5 \%$ impact were excluded). Varying the effectiveness of PrEP and the incidence of HIV in the high-risk group had the greatest impact on ICERs. The results were also sensitive to the costs associated with antiretrovirals for PrEP and in the treatment of HIV. Varying the discount rate had little impact on ICERs.

Owing to the lack of Irish data, much uncertainty exists relating to the size of the target population. We therefore conducted a sensitivity analysis on the proportion of MSM eligible for PrEP (high-risk group). In this analysis, we varied the proportion high risk from $5 \%$ to $50 \%$ (compared with $12 \%$ in the base case). As the proportion of eligible MSM increased, PrEP became more cost saving (see Appendix 6.1 in Supplemental Materials found at https://doi.org/10.1016/j.jval.2021.02.005). Additionally, we carried out a 2-way sensitivity analysis whereby the eligible proportion and the uptake rate were simultaneously varied (from 5\% to $50 \%$ ). ICERs were negatively associated with both variables (see Appendix 6.2 in Supplemental Materials found at https://doi.org/1 0.1016/j.jval.2021.02.005).

We found that the efficacy of PrEP was a significant driver in the model (see Appendix 6.3 in Supplemental Materials found at https://doi.org/10.1016/j.jval.2021.02.005). At all efficacy values 
Figure 2. Incremental cost-effectiveness, PrEP versus no PrEP. Each dot represents an individual simulation of the Monte Carlo analysis. Encircled is the $95 \%$ ellipse. The $x$-axis represents the additional QALY gain per MSM (across all risk groups). The Y-axis represents the incremental cost per MSM (across all risk groups).

\section{Incremental Cost-Effectiveness, PrEP v. No PrEP}

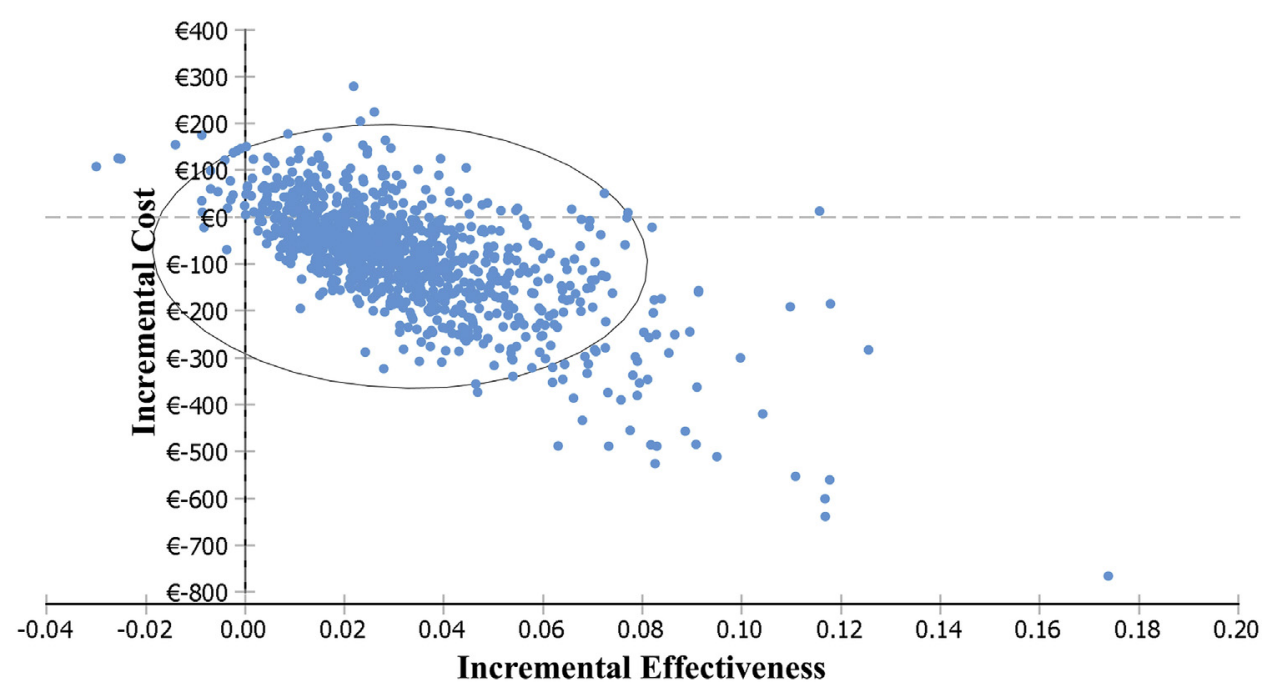

MSM indicates men who have sex with men; PrEP, pre-exposure prophylaxis; QALY, quality-adjusted life-year.

above $60 \%$, we found PrEP to be cost saving. At $44 \%$, the lowest recorded efficacy in MSM (iPrEX trial ${ }^{18}$ ), the ICER was $€ 4711 /$ QALY (highly cost-effective).

\section{Scenario Analysis}

First, we carried out a scenario analysis whereby PrEP users followed event-based dosing. In the only efficacy trial that investigated event-based oral PrEP, a median of 15 pills per month were taken by participants (IPERGAY trial, ${ }^{5}$ which reported an efficacy value identical to daily PrEP). As expected, we found that event-based dosing was associated with additional cost savings, falling to $-€ 4594$, $-€ 5562$, and $-€ 6258 /$ QALY with $50 \%$, 75\%, and $100 \%$ event-based dosing, respectively.

Second, we modeled a scenario that included a baseline PrEP use in the MSM population. In this scenario, we applied an arbitrary baseline PrEP use of 10\% (95\% CI: 5-15) in high-risk MSM to the intervention and comparator groups (whether purchased at community pharmacies or online). We found that the introduction a PrEP program would still be considered cost saving, with little

Figure 3. Tornado diagram (ICER). Blue indicates the effect of increasing the value of the base case and red indicates decreasing the value of the base case. In the base case, the ICER is cost saving.

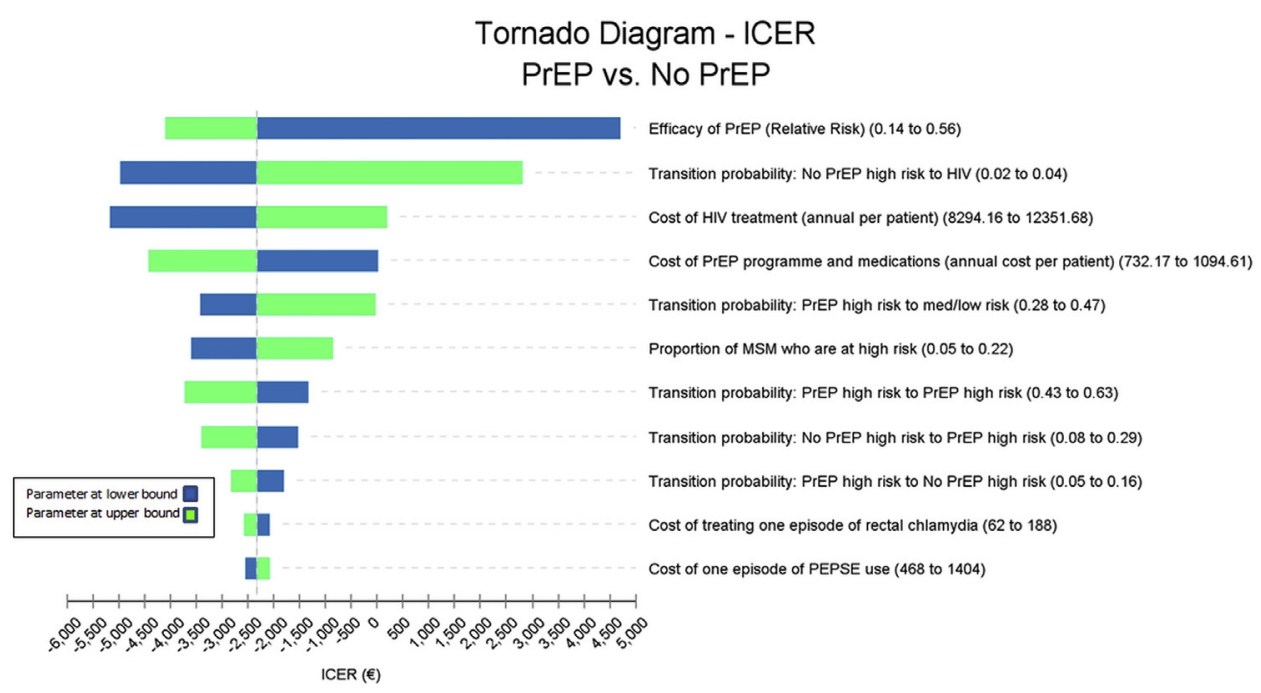


difference in the ICERs. Although the comparator benefitted from a reduction in HIV cases (a proportion of MSM are now using PrEP, without a program in place), there were additional cost savings to the intervention group, because individuals who pay out of pocket incur no costs to the health system.

\section{Discussion}

Our findings showed that PrEP would be considered a costsaving intervention in the first cost-effectiveness analysis of a PrEP program targeted to high-risk MSM in Ireland. We tracked the movement of individuals between risk groups and our time horizon was adequate to capture all costs and consequences accrued over the cohort's lifetime. The main strength of the analysis was its simplicity of design, transparency, and ease of interpretation for policy makers. Our results were robust to considerable variation of parameter values within their plausible ranges as well as variations in the main model assumptions.

The model assumes adequate adherence and correspondingly high clinical effectiveness, as PrEP effectiveness was the main driver of cost-effectiveness in the model. We envisage that PrEP will be delivered as part of a monitored program that includes 3 monthly clinic visits along with medication adherence support and sexual health counseling, which should optimize adherence. The ICERs were also sensitive to the incidence of HIV. Nonetheless, in no scenario investigated did the ICER exceed $€ 5000 / Q A L Y$. We found that ICERs were less sensitive to variations in cost parameters, such as the cost of antiretrovirals for PrEP or in the treatment of HIV (PrEP remained cost saving across a range of plausible values).

Despite the strength of the evidence, of major concern to policy makers is the potential for changes in sexual behavior (risk compensation) in prospective PrEP users and the associated spread of STIs (other than HIV). To date, trial evidence has not demonstrated a rise in STIs associated with PrEP use. However, placebo-controlled trials are not considered appropriate for the assessment of behavior change, because study participants do not know if they are taking PrEP or placebo. The use of observational data to assess risk compensation is also problematic, because studies are often subject to confounding owing to differences in the frequency of STI testing comparing pre- and post-PrEP implementation periods. Taking a conservative approach, we incorporated an increase in STIs in analyses. The inclusion of a rise in STIs had a negligible impact on ICERs. Indeed, the early detection and treatment of STIs in individuals enrolled in a monitored program minimizes the negative consequences and onward transmission associated with STIs.

The comparator in our model was all HIV-negative MSM without access to a PrEP program, whereas in reality, we are aware that some individuals obtain private prescriptions for PrEP and pay out of pocket at community pharmacies, and others obtain PrEP online. We do not know the size of this population, or importantly if these people are truly at high risk and meet the eligibility criteria for PrEP. In any case, when we included a baseline PrEP use in a scenario analysis, PrEP was still considered cost saving.

Prior to this study, we conducted a systematic review of economic evaluations and identified 17 studies relevant to the MSM group. ${ }^{6}$ None were considered applicable to the Irish context, and there was significant variation in parameter values used (for example, the price of daily PrEP ranged from $€ 232$ to $€ 11659$ per patient per year). Many prior studies modeled PrEP over a very short time horizon, failing to capture the lifelong consequences of HIV. $^{28-30}$ Most prior analyses were conducted prior to the availability of generic PrEP, a significantly cheaper alternative to Truvada. $^{31,32}$ In general, our findings are consistent with cost-effectiveness analyses that incorporated generic priced PrEP. Similar to other economic evaluations, ICERs were highly sensitive to adherence-related effectiveness and incidence of HIV. ${ }^{26,33}$

From a health policy and decision-making perspective, the scenario that incorporated event-based dosing is important. Trial data to date have found that event-based PrEP is as effective as daily $\operatorname{PrEP}^{5}$ so there is a potential for significant cost savings without compromising effectiveness. The finding that high PrEP uptake results in additional health benefits and significantly increases cost savings highlights the importance of promoting PrEP within current budgetary constraints.

Our study is subject to a number of limitations. Although the model allowed for the movement of individuals between risk groups over time, individuals in any particular health state were considered a homogenous group independent of prior risk status. Also, for the relationship between high behavioral risk and increased risk of HIV to be valid, the assumption must hold that individuals who are eligible for PrEP are correctly identified. From a health policy perspective, correctly identifying the high-risk group is crucial. Going forward, there may be a growing demand for PrEP in low- and medium-risk groups. Additionally, PrEP users whose risk status changes (to medium/low) may be unwilling to discontinue PrEP. Inappropriate PrEP use in individuals whose risk status is medium or low would reduce the effectiveness and costeffectiveness of the program. The implementation of the program must therefore be based on scientific knowledge on aspects of risk behavior and accompanied by a program evaluation.

The primary limitation of our model's design is that it does not incorporate dynamic elements, which would allow the quantification of the indirect benefits of PrEP on the wider HIV epidemic (as in, the reduction in onward transmission). Our model therefore underestimates the overall population benefit. Dynamic transmission models typically report that the indirect impact of PrEP is modest, however. One dynamic model of the Dutch HIV epidemic found a $13 \%$ to $16 \%$ reduction in ICERs associated with the reduction in onward transmission. ${ }^{34}$

A number of the key parameters used in the probabilistic analysis were subject to significant uncertainty. We derived model parameters from a wide variety of sources, and in situations where there were an absence of reliable data, we calibrated the model to observed data. The application of UK epidemiological data was considered appropriate in some cases, owing to similarities in risk stratification in both jurisdictions (for example, similar eligibility criteria for the provision of PrEP) and broadly comparable HIV incidence and prevalence in the MSM group. It is not yet possible to quantify the uptake rate and size of the PrEP program with any degree of certainty. We therefore used a calibration approach to retrieve plausible estimates for these variables.

Uncertainty also exists relating to HIV care costs, and future reductions in the cost of antiretroviral therapy could significantly reduce the cost of treating HIV. Also, we did not incorporate any indirect costs in our analysis, such as lost productivity owing to HIV. Taking a societal perspective would not change the interpretation of the findings, however, because it would result in PrEP being considered more cost saving.

Going forward, there are 2 model assumptions that must be taken into consideration. First, because we assumed that PrEP will only be taken by individuals at high sexual risk of HIV, regular clinical risk assessment to ensure only those at ongoing risk remain on PrEP is required to maintain cost-effectiveness and ensure equitable access based on clinical need. Second, we assumed that the PrEP program would continue for the cohort's lifetime and that the HIV incidence in each risk stratum would 
remain static over time. Once the incidence and prevalence of HIV decline sufficiently, PrEP may no longer be necessary and may not be considered a cost-effective public health intervention. Although only a small proportion of MSM were administered PrEP in the analysis (2\%), a range of HIV prevention strategies are currently available, and a combination of these interventions are expected to decrease transmission over time.

Since the completion of our study, and in light of our findings, a decision was taken by the Irish government to introduce a publicly funded PrEP program (approving full reimbursement for all eligible individuals). HIV/STI testing, renal monitoring, and clinic visits are also provided free of charge, removing all cost barriers to accessing PrEP. This study highlights the importance of health technology assessment in policy development, and in particular, the power of cost-effectiveness analysis in the decision-making process. Although policy makers and service providers are aware that many interventions are cost-effective, the possibility of actual cost savings through reimbursement decisions are often underappreciated and must be clearly communicated to maximize health benefits and cost savings.

Our findings are likely to be generalizable in countries where cost and epidemiological parameters are comparable, and even if substantially different, our results may still be interpreted with careful consideration of our model assumptions and sensitivity analyses. For example, factors that reduced ICERs in our analysis included increasing the proportion of MSM at high risk, the incidence of HIV, and lifetime costs of HIV. Therefore, jurisdictions that witness a higher prevalence of high-risk behavior and incidence of HIV, or higher HIV care costs, than the Irish MSM population should expect similar findings of cost savings. On the other hand, our analysis is based on generically priced PrEP, and without the availability of generic PrEP the intervention is unlikely to be considered cost saving. Nevertheless, depending on local WTP thresholds, PrEP may still be considered cost-effective.

In conclusion, we found that a publicly funded PrEP program would be considered cost saving in Ireland, and provided significant health benefits to the population through the reduction in HIV transmission.

\section{Supplemental Material}

Supplementary data associated with this article can be found in the online version at https://doi.org/10.1016/j.jval.2021.02.005.

\section{Article and Author Information}

Accepted for Publication: February 10, 2021

Published Online: Month $x x, x x x x$

doi: https://doi.org/10.1016/j.jval.2021.02.005

Author Affiliations: Health Information and Quality Authority, Dublin, Ireland (Murchu, Teljeur, Harrington, Moran, Ryan); Trinity College Dublin, Institute of Population Health, Tallaght, Dublin, Ireland (Murchu, Hayes, Moran); Trinity College Dublin, Department of Pharmacology \& Therapeutics, Trinity Health Sciences, Dublin, Ireland (Ryan).

Correspondence: Eamon O Murchu. Health Information and Quality Authority, George's Court, George's Lane, Dublin 7, Ireland. Email: eomurchu@hiqa.ie

Author Contributions: Concept and design: O Murchu, Teljeur, Hayes, Harrington, Moran, Ryan

Acquisition of data: O Murchu

Analysis and interpretation of data: O Murchu, Teljeur, Hayes, Harrington, Moran, Ryan
Drafting of the manuscript: O Murchu, Teljeur, Hayes, Harrington, Moran, Ryan

Critical revision of the paper for important intellectual content: O Murchu, Teljeur, Hayes, Harrington, Moran, Ryan

Statistical analysis: O Murchu, Teljeur

Supervision: O Murchu, Teljeur, Hayes, Harrington, Moran, Ryan

Conflict of Interest Disclosures: The authors reported no conflicts of interest.

Funding/Support: The authors received no financial support for this research.

Acknowledgment: We would like to thank Dr Fiona Lyons, Dr Susan Clarke, and Dr Saloni Surah for providing epidemiological and cost data from St James's Hospital, Dublin, Ireland. We would also like to thank Ms Siobhan O'Dea (manager) and all the staff at the Gay Men's Health Centre, Dublin, Ireland.

\section{REFERENCES}

1. Health Protection Surveillance Centre (HPSC). HIV in Ireland, 2018. Annual Epidemiological Report; 2019.

2. European Centre for Disease Control and Prevention (ECDC) and the World Health Organization Regional Office for Europe. HIV/AIDS surveillance in Europe - 2018 data. https://www.ecdc.europa.eu/sites/default/files/ documents/HIV-annual-surveillance-report-2019.pdf.

3. Department of Health. Healthy Ireland Survey 2017. http://www.hea lthyireland.ie/wp-content/uploads/2017/10/Healthy-Ireland-Survey-Wave-3Report-1.pdf.

4. McCormack S, Dunn DT, Desai M, et al. Pre-exposure prophylaxis to prevent the acquisition of HIV-1 infection (PROUD): effectiveness results from the pilot phase of a pragmatic open-label randomised trial. Lancet. 2016;387(10013):53-60.

5. Molina J-M, Capitant C, Spire B, et al. On-demand preexposure prophylaxis in men at high risk for HIV-1 infection. N Engl J Med. 2015;373(23):2237-2246.

6. Health Information and Quality Authority (HIQA). Health technology assessment of a PrEP programme for populations at substantial risk of sexual acquisition of HIV. Chapter 5: systematic review of economic evaluations. https://www.hiqa.ie/sites/default/files/2019-06/PrEP-HTA.pdf; 2019.

7. Health Information and Quality Authority (HIQA). Health technology assessment of a PrEP programme for populations at substantial risk of sexua acquisition of HIV. Chapter 6: economic evaluation. https://www.hiqa.ie/ sites/default/files/2019-06/PrEP-HTA.pdf.

8. Health Information and Quality Authority (HIQA). Guidelines for the Economic Evaluation of Health Technologies in Ireland 2018. https://www.hiqa. ie/sites/default/files/2018-01/HIQA_Economic_Guidelines_2018.pdf.

9. EUnetHTA. Methods for health economic evaluations - a guideline based on current practices in Europe. European Network of HTA. https://www. eunethta.eu/wp-content/uploads/2018/01/Methods-for-health-economic-ev aluations-A-guideline-based-on-current-practices-in-Europe_Guideline_Fin al-May-2015.pdf; 2015.

10. TreeAge Pro 2018. TreeAge Software, Williamstown, MA, USA. Available at: https://www.treeage.com/. Accessed April 12, 2021.

11. NHS. Implementation of HIV PrEP in Scotland: first year report. https://www. hps.scot.nhs.uk/resourcedocument.aspx?id=7145. Accessed February 2019. 2019.

12. Grulich AE, Guy R, Amin J, et al. Population-level effectiveness of rapid, targeted, high-coverage roll-out of HIV pre-exposure prophylaxis in men who have sex with men: the EPIC-NSW prospective cohort study. Lancet HIV. 2018;5(11):e629-e637.

13. HSE. Position on antiretroviral therapy for all people living with HIV. https:// www.sexualwellbeing.ie/sexual-health/sexually-transmitted-infections/infor mation-on-hiv/hse-position-on-antiretroviral-therapy_vfeb2018.pdf; 2017. Accessed January 2019.

14. Central Statistics Office. Age-specific mortality by age group, sex, cause of death, year and statistic. https://www.cso.ie/px/pxeirestat/statire/Select VarVal/Define.asp? MainTable=DHA11\&PLanguage=0\&PXSId=0. 2018.

15. O'Donnell K, Fitzgerald M, Barrett P, Quinlan M, Igoe D. MISI 2015: findings from the men who have sex with men internet survey. MSM Internet Survey Ireland. http://www.hpsc.ie/AZ/SpecificPopulations/Menwhohavesexwith menMSM/MISI2015/.

16. Croxford S, Kitching A, Desai S, et al. Mortality and causes of death in people diagnosed with HIV in the era of highly active antiretroviral therapy compared with the general population: an analysis of a national observational cohort. Lancet Public Health. 2017;2(1):e35-e46.

17. Miners A, Phillips A, Kreif N, et al. Health-related quality-of-life of people with HIV in the era of combination antiretroviral treatment: a cross-sectional comparison with the general population. Lancet HIV. 2014;1(1):e32-e40.

18. Grant RM, Lama JR, Anderson PL, et al. Preexposure chemoprophylaxis for HIV prevention in men who have sex with men. $N$ Engl $J$ Med. 2010;363(27):2587-2599. 
19. Grohskopf LA, Chillag KL, Gvetadze R, et al. Randomized trial of clinical safety of daily oral tenofovir disoproxil fumarate among HIV-uninfected men who have sex with men in the United States. J Acquir Immune Defic Syndr. 2013;64(1):79-86.

20. Hosek SG, Siberry G, Bell M, et al. The acceptability and feasibility of an HIV preexposure prophylaxis (PrEP) trial with young men who have sex with men. J Acquir Immune Defic Syndr. 2013;62(4):447-456.

21. Mutua G, Sanders E, Mugo P, et al. Safety and adherence to intermittent preexposure prophylaxis (PrEP) for HIV-1 in African men who have sex with men and female sex workers. PloS One. 2012;7(4):e33103.

22. Ara R, Brazier JE. Populating an economic model with health state utility values: moving toward better practice. Value Health. 2010;13(5):509-518.

23. Health Protection Surveillance Centre (HPSC). HIV in Ireland 2017. Annual epidemiological report. 2017. https://www.hpsc.ie/a-z/hivandaids/ hivdataandreports/2017reports/HIV_2017_report.pdf. Accessed January 2019.

24. Traeger MW, Schroeder SE, Wright EJ, et al. Effects of pre-exposure prophylaxis for the prevention of human immunodeficiency virus infection on sexual risk behavior in men who have sex with men: a systematic review and meta-analysis. Clin Infect Dis. 2018;67(5):676-686.

25. O'Donnell K, Fitzgerald M, Barrett P, Quinlan M, Igoe D. Men who have sex with men Internet Survey Ireland (MISI). 2015.

26. Ong KJ, Desai S, Field N, et al. Economic evaluation of HIV pre-exposure prophylaxis among men-who-have-sex-with-men in England in 2016. Euro Surveill. 2017;22(42), 17-00192.

27. Orr J, McGarrigle C, Kenny RA. Sexual activity in the over 50s population in Ireland. The Irish Longitudinal Study on Ageing. Dublin. 2017.

28. Durand-Zaleski I, Mutuon P, Charreau I, et al. Cost effectiveness of on demand PrEP in men who have sex with men (MSM) in the ANRS IPERGAY study. J Int AIDS Soc. 2016;19:97.
29. MacFadden DR, Tan DH, Mishra S. Optimizing HIV pre-exposure prophylaxis implementation among men who have sex with men in a large urban centre: a dynamic modelling study. J Int AIDS Soc. 2016;19(1):20791.

30. Schneider K, Gray RT, Wilson DP. A cost-effectiveness analysis of HIV preexposure prophylaxis for men who have sex with men in Australia. Clin Infect Dis. 2014;58(7):1027-1034.

31. Juusola JL, Brandeau ML, Owens DK, Bendavid E. The cost-effectiveness of preexposure prophylaxis for HIV prevention in the united states in men who have sex with men. Ann Intern Med. 2012;156(8):541-550.

32. Desai K, Sansom SL, Ackers ML, et al. Modeling the impact of HIV chemoprophylaxis strategies among men who have sex with men in the United States: HIV infections prevented and cost-effectiveness. AIDS. 2008;22(14):1829-1839.

33. Nichols BE, Boucher CAB, van der Valk M, Rijnders BJA, van de Vijver D. Costeffectiveness analysis of pre-exposure prophylaxis for HIV-1 prevention in the Netherlands: a mathematical modelling study. Lancet Infect Dis. 2016;16(12):1423-1429.

34. Nichols BE, Boucher CA, Van Der Valk M, Rijnders BJ, Van De Vijver DA. Prep is only cost-effective among MSM in the Netherlands when used on demand. Top Antivir Med. 2016;24(E-1):456.

35. Department of Health. Healthy Ireland Survey 2015. Available at: https:// www.gov.ie/en/publication/fedbc9-healthy-ireland-survey-2015-summaryof-findings/. Accessed April 12, 2021.

36. Department of Health and the HSE Sexual Health and Crisis Pregnancy Programme. The Irish Study of Sexual Health and Relationships, 2006. Available at: https://www.ucd.ie/issda/static/documentation/esri/isshrreport.pdf. Accessed April 12, 2021.

37. Nakagawa Fumiyo, Miners Alec, Smith Colette J, et al. Projected lifetime healthcare costs associated with HIV infection. PLoS One. 2015;10(4): e0125018. 